\title{
A NOTE ON SEPARABLE BANACH SPACES WITH NONSEPARABLE DUAL
}

JAMES N. HAGLER

\begin{abstract}
If $X$ is a separable Banach space with $X^{*}$ nonseparable, then $X$ contains a subspace $X_{0}$ with a Schauder basis with $X_{0}^{*}$ nonseparable.
\end{abstract}

Introduction. At the Kent State conference on Banach spaces and classical analysis in August 1985, V. Zizler asked if a separable Banach space with nonseparable dual had a subspace with a Schauder basis with the same property. In this note we give an affirmative answer to this question by modifying the well-known construction of Stegall [STE]. Stegall showed that if $X$ is separable and $X^{*}$ is not, there is a procedure for constructing a sequence $\left(x_{n}\right)$ in $X$ which, among its additional properties, spans a subspace with a nonseparable dual. What is shown here is that this sequence can be chosen in such a way that it is also a Schauder basis.

Following the recent trend in discussions of Stegall's result (which is notationally quite messy), we give only the first few steps of the construction, and leave it to the reader to fill in the remaining details.

Our notation is standard and undefined notation and concepts are described either in the book of Day [DAY] or of Lindenstrauss and Tzafriri [L-T]. The first uncountable ordinal is denoted by $\omega_{1}$. Also, if $E$ is a Banach space, then $S_{E}=\{e \in$ $E:\|e\|=1\}$ is the unit sphere of $E$ and $B_{E}=\{e \in E:\|e\| \leqslant 1\}$ is the unit ball of $E$.

Our first lemma is an obvious extension of Lemma 1 of [STE].

Lemma. Let $X$ be separable, $X^{*}$ nonseparable, $Y$ a separable subspace of $X^{*}$, and $\varepsilon>0$. Then there exist families $\left\{f_{\alpha}: \alpha<\omega_{1}\right\}$ in $S_{X^{*}}$ and $\left\{G_{\beta}: \beta<\omega_{1}\right\}$ in $(1+\varepsilon) B_{X^{* *}}$ such that

(i) $G_{\beta \mid Y}=0$ for all $\beta$.

(ii)

$$
G_{\beta}\left(f_{\alpha}\right)= \begin{cases}1 & \text { if } \beta=\alpha \\ 0 & \text { if } \beta>\alpha .\end{cases}
$$

(iii) Every point of $\left\{f_{\alpha}: \alpha<\omega_{1}\right\}$ is a weak ${ }^{*}$ condensation point of $\left\{f_{\alpha}: \alpha<\omega_{1}\right\}$.

We use Lemma 1 where $Y$ is a separable subspace of $X^{*}$ which isometrically norms $X$, i.e. for each $x \in X$,

$$
\|x\|=\sup \{y(x): y \in Y,\|y\|=1\} .
$$

Received by the editors November 8, 1985.

1980 Mathematics Subject Classification (1985 Revision). Primary 46B10, 46B15. 
THEOREM. If $X$ is a separable Banach space with nonseparable dual $X^{*}$, there exists a basic sequence $\left(x_{n}\right)$ with $\left[x_{n}\right]^{*}$ nonseparable. (The closed linear span of the sequence $\left(x_{n}\right)$ is denoted $\left[x_{n}\right]$.)

Proof. The idea of the proof is to repeat Stegall's argument word for word with an additional finite number of weak* conditions at each stage which force the constructed sequence $\left(x_{n}\right)$ to be a basic sequence. Rather than using Stegall's notation, we follow that used in Diestel and Uhl [D-U, pp. 192-194]. To obtain a basic sequence, we use the well-known Mazur construction (see Diestel [D, pp. 32-43] for a discussion).

Let $Y \subset X^{*}$ be a separable, isometrically norming subspace, let $\left\{f_{\alpha}\right\},\left\{G_{\beta}\right\}$ be as in the lemma, and let $\varepsilon>0$. Let $\varepsilon_{n} \rightarrow 0$ such that $\Pi\left(1-\varepsilon_{n}\right)^{-1}<1+\varepsilon$.

By Helly's theorem [DAY, Corollary 2, p. 39] select $x_{1},\left\|x_{1}\right\|<1+\varepsilon$ with $G_{1}\left(f_{1}\right)=f_{1}\left(x_{1}\right)=1$. Put $W_{1}=\{y\}$ where $y \in Y$ satisfies $\|y\|=1$ and $y\left(x_{1}\right) \geqslant$ $\left(1-\varepsilon_{1}\right)\left\|x_{1}\right\|$. Put $A_{1}=\left\{\alpha: f_{\alpha}\left(x_{1}\right)>1-\varepsilon\right\}$. This completes the selection of $x_{1}$.

We now turn to the construction of $x_{2}$ and $x_{3}$. Since $A_{1}$ is uncountable, we can choose $\beta_{1}, \alpha_{2} \in A_{1}$ with $1<\beta_{1}<\alpha_{2}$. Then

(i) $G_{\alpha_{2}}\left(f_{\alpha_{2}}\right)=1$,

(ii) $G_{\alpha_{2}}\left(f_{\beta_{1}}\right)=0$,

(iii) $G_{\alpha_{2}}(y)=0$.

By Helly's theorem, there is an $x_{2},\left\|x_{2}\right\|<1+\varepsilon$, with

(i) $f_{\alpha_{2}}\left(x_{2}\right)=1$,

(ii) $f_{\beta_{1}}\left(x_{2}\right)=0$,

(iii) $y\left(x_{2}\right)=0$,

It follows that if $\lambda \in \mathbf{R},\left\|x_{1}+\lambda x_{2}\right\| \geqslant y\left(x_{1}+\lambda x_{2}\right)=y\left(x_{1}\right) \geqslant\left(1-\varepsilon_{1}\right)\left\|x_{1}\right\|$.

Now let $Z_{2}$ be a finite $\varepsilon_{2} / 2$ net in $\left\{x \in\left[x_{1}, x_{2}\right]:\|x\|=1\right\}$, and let $W_{2}$ be a finite subset of $\{y \in Y:\|y\|=1\}$ such that for each $z \in Z_{2}$ there is a $y \in W_{2}$ with $y(z)>1-\varepsilon_{2} / 2$.

Since $\beta_{1}$ is a condensation point of $A_{1}$, there exists $\alpha_{3}>\alpha_{2}$ such that $f_{\alpha_{3}}\left(x_{2}\right)<$ $\varepsilon / 4$. Observe that the following finite number of conditions are satisfied:

(i) $G_{\alpha_{3}}\left(f_{\alpha_{2}}\right)=0$,

(ii) $G_{\alpha_{3}}\left(f_{\alpha_{3}}\right)=1$,

(iii) $G_{\alpha_{3}}(y)=0$ for all $y \in W_{2}$.

Applying Helly's theorem once again, we find an $x_{3} \in X,\left\|x_{3}\right\|<1+\varepsilon$ such that

(i) $f_{\alpha_{2}}\left(x_{3}\right)=0$,

(ii) $f_{\alpha_{3}}\left(x_{3}\right)=1$,

(iii) $y\left(x_{3}\right)=0$ for all $y \in W_{2}$.

Let $x \in\left[x_{1}, x_{2}\right],\|x\|=1$, and let $\lambda \in \mathbf{R}$. Pick $z \in Z_{2}$ such that $\|z-x\|<\varepsilon_{2} / 2$ and pick $y \in W_{2}$ with $y(z)>1-\varepsilon_{2} / 2$. Then

$$
\begin{aligned}
\left\|x+\lambda x_{3}\right\| & \geqslant y(x)-\lambda y\left(x_{3}\right) \geqslant y(z)-|y(z-x)| \\
& \geqslant 1-\varepsilon_{2} / 2-\varepsilon_{2} / 2 \geqslant\left(1-\varepsilon_{2}\right)\|x\| .
\end{aligned}
$$

Now continue the construction inductively as in [D-U, pp. 192-193]. The new part of the construction (namely the use of the finite, almost-norming sets $W_{i}$ ) forces the 
norms of the coordinate projections $P:\left[x_{1}, \ldots, x_{m}\right] \rightarrow\left[x_{1}, \ldots, x_{n}\right](m \geqslant n)$ given by

$$
P\left(\sum_{i=1}^{m} \lambda_{i} x_{i}\right)=\sum_{i=1}^{n} \lambda_{i} x_{i}
$$

to be uniformly bounded by $1+\varepsilon$. The other part of the construction is identical to Stegall's construction and forces the sequence $\left(x_{n}\right)$ to span a space with a nonseparable dual. This completes the proof.

Added IN Proof. The main result of this paper has also been obtained by $\mathrm{H}$. Rosenthal [ Weak* polish Banach spaces (to appear)].

\section{REFERENCES}

[D] J. Diestel, Sequences and series in Banach spaces, Graduate Texts in Math., no. 92, Springer-Verlag, New York, 1984.

[DAY] M. M. Day, Normed linear spaces, 2nd printing, Springer-Verlag, New York, 1962.

[D-U] J. Diestel and J. J. Uhl, Vector measures, Math. Surveys, no. 15, Amer. Math. Soc., Providence, R. I., 1977.

[L-T] J. Lindenstrauss and L. Tzafriri, Classical Banach spaces. Vol. I. Sequence spaces, Springer-Verlag, New York, 1977.

[STE] C. Stegall, The Radon-Nikodym property in conjugate Banach spaces, Trans. Amer. Math. Soc. 206 (1975), 213-223.

Department of Mathematics and Computer Science, University of Denver, Denver, ColORADO 80208 